\title{
Anchor Date
}

National Cancer Institute

\section{Source}

National Cancer Institute. Anchor Date. NCI Thesaurus. Code C160815.

The base date from which all other dates within a cohort or data set are derived. 